\title{
Magnetic Field Structure of the CME Source Region
}

\author{
Y. Hanaoka \\ National Astronomical Observatory of Japan, 2-21-1 Osawa, Mitaka, \\ Tokyo 181-8588, Japan
}

\begin{abstract}
The three-dimensional structure of the magnetic field in the source region of CMEs is the key to understand how the stored magnetic energy eventually causes an eruption. A CME accompanied by a filament eruption on 2000 February 26-27 is particularly a good event to study the three-dimensional magnetic field structure. This event was very well observed with the EIT and LASCO of SOHO and the SXT of Yohkoh, and shows the following clues of the magnetic field structure which caused the CME. (1) The filament had a helical structure before the eruption and it was kept throughout the eruption. (2) The coronal loop structure shows that this event was an eruption of a part of the quadrapolar magnetic field structure consisting of two active regions. In this proceeding, we present a brief overview of the event.
\end{abstract}

\section{Introduction}

CMEs are sometimes spectacular events and recently their behaviors are beautifully shown by the LASCO of SOHO. However, the answer to the key question about the mechanism of the CME initiation is still unclear. The essential change from the quasi-static state before the eruption to the erupting stage is basically the change of the magnetic field in the source region of the CMEs in the low corona. Therefore, the three-dimensional magnetic field structure of the low corona is essentially important to study how the energy of an eruption is stored, and how an eruption is initiated. Even though the currently available sophisticated instruments give a new insight into the study of the CME/filament eruption (e.g., Dere et al. 1997; Innes et al. 1999), generally it is not easy to observe both the structure of the coronal loops and the photospheric magnetic field where the coronal loops are rooted at the same time. The best events to study the three-dimensional structure are the eruptions occurring near, but not very close to, the limb. In such a case, most part of the coronal loops in the CME source region is seen above the limb without contamination from the bright low-lying corona. On the other hand, photospheric magnetic field can be observed, even though the measurement undergoes the projection effect.

In this paper, we present a case study of a CME, which took place on 2000 February 26-27. The CME is accompanied by an eruption of a filament, which was about 60 degrees away from the disk center, and therefore, this event is one of the best event to study the CME source region. The evolutions of both the $\mathrm{CME}$ and the filament eruption was tracked from the solar surface to over 10 

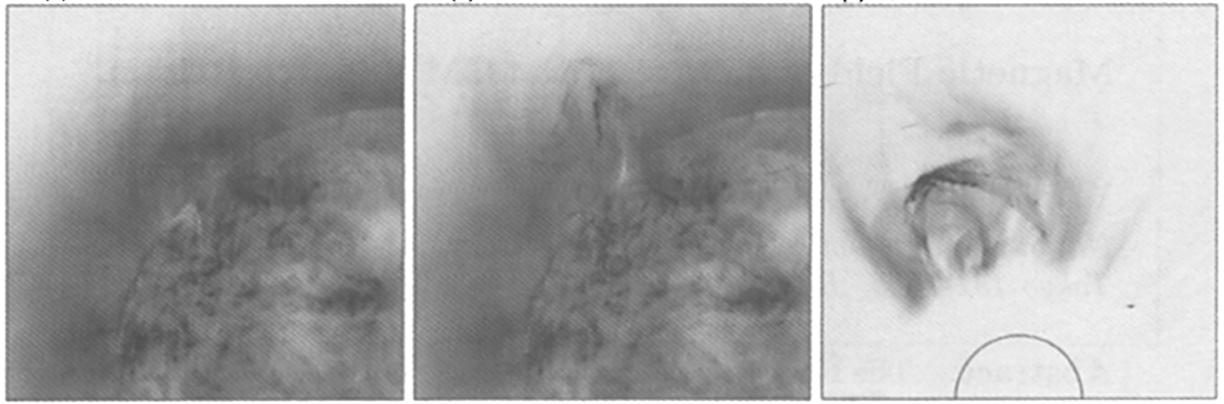

(d) SXT Al0.1 26-Feb-2000 21:59:12

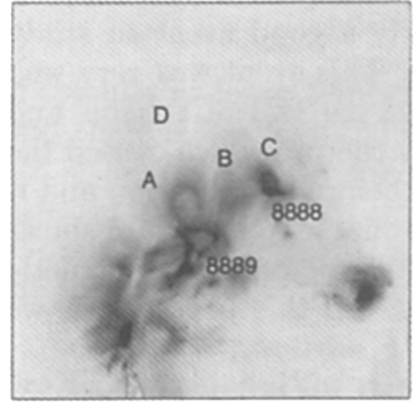

(e)
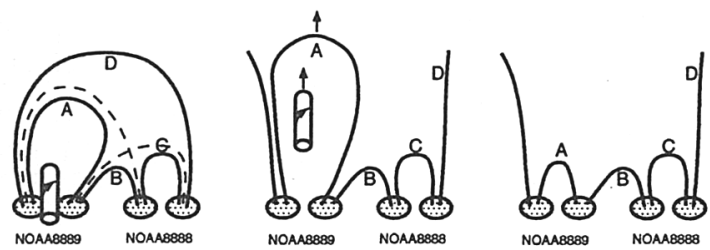

Figure 1. CME and filament eruption observed with (a-b) the EIT and (c) the LASCO of SOHO. (d) The coronal loop structure observed with the SXT of Yohkoh just before the eruption. All images are shown in negatives. (e) Schematic drawing of the filament and the coronal loops in the CME source region before, during, and after the eruption.

solar radii with the EIT and LASCO of SOHO and the SXT of Yohkoh. Because of the page limitation, here we just present a brief overview of the event, and the detailed discussion will appear in a forthcoming paper.

\section{The CME on 2000 February 26-27}

\subsection{Overview}

The eruption of the corona and the filament on 2000 February 26-27 observed with the EIT and the LASCO of SOHO is shown in Figures 1a-c. Figure 1a, an image in a cool coronal line Fe XII $195 \AA$ taken before the eruption with the EIT, shows a dark filament and overlying loop structure. Soon after the gradual start of the ascending motion of the filament and the expansion of the coronal loop, the eruption proceeded abruptly (Figure 1b). About 1.5 hours after the start of the abrupt eruption, a CME frontal loop and the filament inside are clearly seen in the field of view of the LASCO C2 (Figure 1c). Naturally this structure is considered as the expanded filament and overlying coronal loop seen in the EIT images. 
The ascending speed of the CME front is about $700 \mathrm{~km} \mathrm{~s}^{-1}$, and that of the filament is $460 \mathrm{~km} \mathrm{~s}^{-1}$.

\subsection{Helical Structure of the Filament}

In this event, a helical structure of the filament was seen from the solar surface through about $10 R_{\odot}$. In Figure 1b, taken just after the beginning of the abrupt eruption, a 'right-helical' structure of the erupting filament is easily found. It shows about two turns from one end of the filament to the other. The filament before the eruption (Figure 1a and $\mathrm{H} \alpha$ pictures) also shows a helical structure. Therefore, the helicity did exist before the eruption, and it is presumed that the magnetic field including such a helical structure contributes to the eruption. The filament observed afterward with the LASCO (Figure 1c) looks like a twisted blade. The series of images taken with the LASCO show a homologous expansion of such a structure. The filament seen in the LASCO images shows about one turn, but they show only upper part of the filament. Therefore, this is probably a part of the twisted filament seen in the images taken with the EIT. Therefore, we can conclude that the twisted filament erupted and it basically kept its helical structure during the eruption, without a substantial untwisting.

\subsection{Coronal Loop Structure in the CME Source Region}

Figure 1d shows the coronal structure before eruption in soft X-rays observed with the SXT of Yohkoh. The filament is located just to the north of region NOAA 8889, and the overlying loop of the filament is labelled ' $A$ ' in Figure 1d. The soft X-ray images indicate that the loop overlying the filament is a part of a system of quadruple loops, which consists of regions NOAA 8889 and 8888 . A sketch of its structure through the eruption is shown in Figure 1e. The overlying loop of the filament, loop A has a balloon-like shape before the eruption. Such a structure is clearly different from a presumed potential field structure based on MDI magnetograms, while the post-flare loops seem to be potential-like. The structure of the loop system seen in Figures 1d and 1e and the helical structure of the filament described in the previous subsection is the observational structure of the magnetic field in the CME source region, which is about to erupt. Detailed comparison of such observational results with models proposed by, e.g., Kuperus and van Tend (1981) and Kusano et al. (1995) and discussion about energetics of such a loop structure will be described in a forthcoming paper.

\section{References}

Dere, K. P. and 33 co-authors, 1997, Solar Phys. 175, 601

Innes, D. E. and 8 co-authors, 1999, Solar Phys. 186, 337

Kuperus, M. and van Tend, W. 1981, Solar Phys. 71, 125

Kusano, K., Suzuki, Y., and Nishikawa, K. 1995, ApJ, 441, 942 